\title{
Some Significant Trends in Textile Bleaching
}

\author{
N. Gokarneshan*
}

\section{Department of Textile Technology, Park College of Engineering and Technology, Coimbatore, India}

\begin{abstract}
The article highlights some recent trends in the bleaching of textile materials. An attempt has been made to compare the effect of electrochemical bleaching with conventional bleaching of cotton. Electric current is used in the preparation of a bleaching agent to replace bleaching powder. The sodium hypochlorite generated from electrolyte cell is sufficient to produce powerful bleaching agent with available chlorine. Electrochemical bleaching with sodium hypochlorite offers numerous advantages like prevention of effluent problem, economy, reliability, cleanliness, and convenience in working. Attempts have been made to use sodium perborate as a bleaching agent with potassium persulphate as an activator at lower temperature in combined pre treatment of cotton fabric. Experimental trials have been designed by using taguchi technique. This bleaching technique promises to be eco-friendly process. In yet another interesting work, effort has been taken to bleach the scoured cotton fabric with sodium perborate as bleaching agent and tetreacetyl ethylenediamine as bleaching activator. The concentrations of these chemicals, temperature and time of the treatment have been varied. The utilization of hydrogen peroxide is much higher as compared with that of conventional bleaching process. Other advantages include less requirement of water and energy, thereby satisfying needs of ecofriendly process, lesser loss in weight, tearing strength, and tensile strength, in comparison with conventional bleaching process without compromising whiteness index.
\end{abstract}

Keywords: Sodium hypochlorite, Electrochemical bleaching, tetreacetyl ethylenediamine, Whiteness index, Cotton fabric, Combined pretreatment.

\section{INTRODUCTION}

Bleaching is a process by which natural colouring matter and any other colouring matter is removed from natural fibres or man made fibres or also known as a process delolourisation [1]. If the fibre brightness is not important, no bleaching is required. However, for light and medium shades or when fibre brightness is important, bleaching becomes an essential operation before dyeing and printing. The natural colouring matter can be destroyed by oxidation or by reduction or as a combination of both [2].

The colour producing agents in natural fibres are often organic compounds containing conjugated double bonds. Decolouration can occur by breaking up the chromopore, most likely destroying one or more of the double bonds within the conjugated system [3]. There are two types of bleaching agents - reducing and oxidizing. Due to the concern for eco friendliness, oxidative bleaching using hydrogen peroxide is practiced on a large scale commercially.

Today's textile industry is very much aware of conserving energy in wet processing for eco friendly approach as well as for increasing the profit margins. Hydrogen peroxide bleaching is beneficial only when it is either carried out at low temperature or in as minimum time as possible. In conventional peroxide

*Address correspondence to this author at the Department of Textile Technology, Park College of Engineering and Technology, Coimbatore, India; E-mail: advaitcbe@rediffmail.com bleaching, efficient bleaching of peroxide requires higher temperature since at lower temperature it becomes ineffective [4].

\section{ELECTROCHEMICAL BLEACHING OF COTTON}

Oxidative bleaching is preferred over reductive bleaching because whiteness produced by a reducing agent is not permanent [5]. Out of all the bleaching agents, bleaching with sodium hypochlorite or hydrogen peroxide is widely used. Nowadays, the problem associated with sodium hypochlorite bleaching is encouraging the industrialist to opt for hydrogen peroxide bleaching [6]. But electrochemically sodium hypochlorite can be generated and used as a bleaching agent without transportation or storage of hypochlorite liquor. Sodium hypochlorite has been generated in situ with a simple electrolytic cell having a potential difference inside the cell of 2.5-3 $\mathrm{V}$ and the effect of electrochemical bleaching is compared with conventional bleaching of cotton. There is also no effluent problem associated with electrochemical bleaching. It is also advantageous in respect of economy, reliability, cleanliness and convenience in working.

The electrolyte cell is charged with $30 \mathrm{~g} / \mathrm{L}$ of salt solution and fitted with two carbon plate electrodes, the anode being covered with red calico. In the electrolytic decomposition of sodium chloride salt the chloride is separated into its constituent elements the chloride is separated, sodium and chlorine, the former combining with water to form sodium hydroxide and hydrogen. 
Simultaneous presence of chlorine and sodium hydroxide give sodium hypochlorite. In the electrolyte the following reactions take place

$2 \mathrm{Na}^{+}(\mathrm{aq})+\mathrm{Cl}_{2}(\mathrm{~g})+2 \mathrm{OH}(\mathrm{aq})--\rightarrow \mathrm{NaOCl}(\mathrm{aq})+\mathrm{NaCl}(\mathrm{aq})$ $+\mathrm{H}_{2} \mathrm{O}$

The raw materials used in electrolytic cell have been specified [6-8].

The whole study is divided into two parts, namely,

a) Preliminary study intended to determine the optimum conditions for electrolysis, and

b) Comparative study between electrochemical and conventional bleaching process

In the preliminary study, electrolysis has been carried out by varying the concentration of the solution $(10,25,50,70$ and $100 \mathrm{~g} / \mathrm{L})$ iodometric titrations have been carried out using potassium iodide, sodium bi sulphite, glacial acetic acid and starch as reagents. Available chlorine is calculated after 70 minutes by using the formula $[7,8]$

$\%$ Active chlorine $=\left[3.545^{*} \mathrm{~N}^{*} \mathrm{~V}\right] / \mathrm{Y}$

Where $\mathrm{V}$ is the volume of $0.1 \mathrm{~N}$ sodium bi sulphite consumed; $\mathrm{Y}$, the volume of the sample taken, and $\mathrm{N}$ the normality of the potassium iodide solution.

It is concluded that the industrial feasibility of a process needs various considerations. It must fulfil various protocols and industry requirements and must comply with the most stringent environmental rules. These factors have been studied on a pilot scale. Optimized conditions have been sought and a number of conclusions drawn. There is material saving resulting in less consumption of chemicals. Only AR grade salt and water can produce the required sodium hypochlorite. The electrodes used are also cheap and have long life. Maintenance cost is also very less. Better whiteness index has been achieved. It is observed that the average whiteness index of the electrochemically bleached samples is greater than that of the conventionally bleached, scoured and grey samples. The average yellowness index of the electrochemically bleached samples is less and they have higher bleaching index than the other samples. The process reliability is very good. The in situ manufacturing of sodium hypochlorite is highly reliable because this need not depend on the suppliers for the chemicals. Sudden increase in the cost of the chemicals will not affect the process house [9]. There is shorter consumption of time. In conventional bleaching it takes around 90 minutes to bleach a sample properly, but in the electrochemical bleaching it takes hardly 10 20 minutes to bleach the sample after attainment of the required concentration of available chlorine. Thus there is a large saving of time. It is a clean process. The electrochemical bleaching is clean and thus the working in this environment can be more pleasant for the persons. The process is eco-friendly. Electrochemical bleaching is eco-friendly because there is no production of water polluting chemicals. Thus, there is no effluent problem.

These results offer new prospects for the electrochemical bleaching. This innovative electrochemical bleaching of cotton can usher a new era in textile processing industry with further applications and markets to be explored.

\section{USE OF SODIUM PERBORATE AND POTASSIUM PERSULPHATE}

In conventional processing, every pretreatment such as desizing, scouring, and bleaching is performed separately. Each pretreatment has to be followed by thorough washing to remove the impurities of the process and the extraneous chemicals causing large consumption of water and energy in addition to the time required to obtain bleached fabric. Conventional hydrogen peroxide bleaching is carried out at $85-90^{\circ} \mathrm{C}$ for 4 hours at alkaline $\mathrm{pH}$ between 10 and 11 using sodium silicate as a stabilizer. Sodium silicate is non eco-friendly and it gives harsh feel to the bleached cotton material. The consumption of water is about $40 \mathrm{~L} / \mathrm{kg}$, which is considerably high. The requirement of energy in the form of steam is also more $(205 \mathrm{~kg} / \mathrm{kg}$ of fabric) [10]. Due to rapid increase in cost of energy, the research is focussed on low temperature applications or combining the pre treatment processes.

Combined scouring and bleaching process is now more common for cotton and cotton blends. But whiteness may be little inferior than sequential scouring and bleaching process. Time required for combined process is nearly 4-6 hours in batch process. Time required for combined process is nearly 4-6 hours in batch process.

Sodium perborate is a white, odourless, water soluble powder. The monohydrate and tetrahydrate are the commercially important forms, of which the former dissolves better and has higher heat stability. Sodium 
perborate undergoes hydrolysis in contact with water, producing hydrogen peroxide and borate.

Potassium persulphate has been used as an activator in bleaching with potassium perborate. The effect of temperature, time and concentrations of sodium perborate, potassium persulphate and caustic soda on whiteness of treated fabrics is studied by using taguchi analysis. The quality of bleached fabric is assessed by evaluating whiteness index, strength loss, carboxyl group content and copper number of the treated cotton and the results are compared with conventional combined pre-treatment process.

The processing sequence for conventional bleaching process is as follows:

Mill grey fabric --- $\rightarrow$ Conventional bleaching and scouring $---\rightarrow$ Hot wash $----\rightarrow$ Cold wash $-\rightarrow$ Cold wash.

The processing sequence for modified bleaching process is as follows

Mill grey fabric --- $\rightarrow$ Modified scouring and bleaching -$--\rightarrow$ Hot wash $----\rightarrow$ Cold wash $--\rightarrow$ Cold wash.

Sodium perborate undergoes hydrolysis in the presence of water, producing hydrogen peroxide and borate as indicated in the following reaction [11]:

$\mathrm{NaBO}_{3} \cdot 4 \mathrm{H}_{2} \mathrm{O} \rightarrow \mathrm{NaBO}_{2}+\mathrm{H}_{2} \mathrm{O}_{2}+3 \mathrm{H}_{2} \mathrm{O}$

(Sodium perborate tetrahydrate) (Sodium metaborate) (Hydrogen peroxide)

In order to determine the optimum experimental configuration of design parameters for performance, quality and cost, taguchi analysis has been used which is systematic and efficient

Combined scouring and bleaching process is now more common for cotton and cotton blends. But whiteness may be little inferior than sequential scouring and bleaching process. Time required for combined process is nearly 4-6 hours in batch process.

The modified combined pre-treatment process using potassium persulphate as an activator along with sodium perborate as an oxidising agent has proved to be efficient in giving results at par with the conventionally combined pre-treatment process, wherein peroxide is used as bleaching agent. Modified process can conserve $50 \%$ energy and time as well as $30 \%$ saving of water [12]. Also, the effluent load is reduced as compared to conventional combined bleaching process. It can be concluded that in the modified process, the utilization of active species that are responsible for bleaching action is better as compared to conventional peroxide bleaching.

\section{ROLE OF TETRAACETYL ETHYLENEDIAMINE IN COTTON BLEACHING WITH SODIUM PERBORATE}

Low temperature bleaching with hydrogen peroxide is possible only by producing peracetate anion. However, this reaction is corrosive and also not stable. Tetraacetyl ethylenediamine is being used as a bleach activator in the laundry with a detergent so that bleaching can be carried out at low temperature [13]. Tetraacetyl ethylenediamine activated bleaching system can be used for cellulosic textiles for improved quality.

Sodium perborate is a white, odourless, water soluble powder. It undergoes hydrolysis in contact with water, producing hydrogen peroxide and borate [14]. Reaction of hydrogen peroxide, water and tetraacetyl ethylenediamine releases per acid or peroxy acid, which is very strong oxidant [15]. The redox potential value is nearly $2.3 \mathrm{~V}$, whereas aqueous mixture of peroxide is giving redox potential of $1.8 \mathrm{~V}$.

Sodium perborate has been used as an oxidising agent with tetraacetyl ethylenediamine as an activator. The effect of temperature, time, and concentrations of sodium perborate and tetraacetyl ethylenediamine on whiteness of treated fabrics has been studied by using taguchi analysis. The quality of bleached fabric is assessed by evaluating whiteness index, strength loss, carboxyl group content, and copper number of treated cotton and the results are compared with conventional bleaching process. Also, the residual hydrogen peroxide concentrations of both bleaching baths are evaluated.

The modified bleaching process using tetraacetyl ethylenediamine as an activator along with sodium perborate as an oxidising agent has proved to be efficient in giving results at par with the conventional peroxide bleaching process [16]. Modified process can conserve $55 \%$ energy and time as well as $30 \%$ saving of water since maximum whiteness is observed at $70^{\circ} \mathrm{C}$ and 2 hours treatment time as compared to conventional peroxide bleaching carried out at $90^{\circ} \mathrm{C}$ for 4 hours. Also, the effluent load is reduced due to elimination of sodium silicate stabilizer and reduced number of washings that are required in combined 
bleaching process. Thus, it can be concluded, that in the modified process, the utilization of active species (hydrogen peroxide) that is responsible for bleaching action is better as compared to that in conventional bleaching.

\section{CONCLUSION}

The influence of electrochemical bleaching of cotton has been compared with that of conventional method. Time required for bleaching reduces to 10-20 minutes, as compared to 90 minutes in conventional bleaching. Whiteness index, brightness and yellowness index have been measured by a spectrophotometer. There is a remarkable increase in whiteness index and brightness index and decrease in yellowness index in the electrochemically bleached samples as compared to that in conventionally bleached cotton. Dye uptake values show that an increasing trend with electrochemical bleaching. It has been aimed to achieve the desired efficiency to remove conventional drawbacks of sodium hypochlorite bleaching. In using sodium perborate as a bleaching agent with potassium persulphate as activator, experimental trials have been designed by using taguchi technique. The results obtained by using this modified bleaching process are compared with the conventional combined pre treatment process. The findings indicate conservation of energy by $50 \%$ and that of water and process time by $30 \%$. The whiteness index is also found to be higher than conventional combined pre treatment process. Thus, the modified process fulfils the requirements of eco-friendly concept. In bleaching of scoured cotton fabric with sodium perborate as bleaching agent and tetraacetyl ethylenediamine as activator the concentrations of the chemicals, temperature and time of the treatment have been varied. When sodium perborate comes in contact with water in bleaching bath, it releases hydrogen peroxide which is responsible for bleaching action on cotton. The findings of this novel process on the quality of bleached fabric are compared with the fabric bleached conventionally using only hydrogen peroxide. The results show that the utilization of generated hydrogen peroxide reaches as high as $96 \%$ in the modified bleaching process, which is only $70 \%$ in the conventional bleaching. Further, the modified bleaching process requires less water and energy, thus fulfilling the requirements of eco-friendly process. Loss in weight, tearing strength, and tensile strength are found to be less in comparison with conventional bleaching without compromising with whiteness index. CIE whiteness with conventional bleaching is 57 , whereas modified recipe shows whiteness.

\section{REFERENCES}

[1] Shenai VA. Technology of bleaching, Sevak publication 1975; 3: 225.

[2] Trotman ER. The bleaching and dyeing of textile fibres, John Wiley and sons Inc., 1975; 216.

[3] http://www.slideshare.net/aleya/bleaching-textile-treatment35250805.

[4] Karmakar SR. Chemical technology in the pretreatment processes in Textiles 1999; 12: 160.

[5] Marsh JT. An introduction to textile bleaching, John wiley and sons, Inc., 1964; 215.

[6] Kapil PN. Physical chemistry, S.Dinesh \& Co., 2008; 1: 112.

[7] Jauhar SP. ABC of Chemistry, Modern Publication 2008; 96.

[8] John E Nettles. Handbook of chemical specialities, John Wiley \& Sons Inc., 1984; 428.

[9] Das D, Patra AK, Jakhar R, Shyam S. Electrochemical bleaching of cotton. Indian Journal of Fibre \& Textile Research 2016; 41: 217.

[10] Wasif Al, Yindi YM. Indian Journal of Fibre and Textile Research 2010; 35: 353.

[11] Yuskel GY, Titiz S, Bulutsu AN. Journal of Chemical Engineering Data 1996; 41: 586. https://doi.org/10.1021/je950267r

[12] Yashovardhan MI, Wasif A, Patel AA. Activated bleaching with sodium perborate and potassium persulphate. Indian Journal of Fibres and Textile Research 2017; 42: 235.

[13] Prabhaharan M, Almeida L. Indian Journal of Fibres and Textile Research 2004; 29(3): 343.

[14] Mathews J. Industrial and Engineering Chemistry 1911; 3(3): 141.

[15] Hu QY, Daneault C, Robert S. Cellulose Chemical Technology 2011; 45: 381.

[16] Yashovardhan MI, Wasif A. Sodium perborate bleaching of cotton by using tetraacetyl ethylenediamine activator. Indian Journal of Fibres and Textile Research 2018; 43: 120. 